\title{
El estrés postraumático como precursor de daños en salud mental y cognición en víctimas de violencia*
}

\section{Post-Traumatic Stress as a Precursor of Mental Health Damage and Cognitive Impairment in Victims of Violence}

\author{
Edna Johanna Herrera Merchán** \\ ORCID: 0000-0002-1002-4044 \\ Universidad Manuela Beltrán \\ Dora Cristina Cañas Betancur*** \\ ORCID: 0000-0003-2954-1241 \\ Universidad Cooperativa de Colombia \\ Recibido: 21 de noviembre de 2019 \\ Revisado: 7 de febrero de 2020 \\ Aceptado: 12 de abril de 2020
}

\section{Resumen}

Este artículo tiene como objetivo mostrar como la violencia es un acto dañino a nivel social que genera detrimento en la salud mental y deterioro cognitivo. Se realizó una revisión sistemática basada en una estrategia de búsqueda siguiendo los lineamientos PRISMA. Estos artículos estuvieron relacionados con la psicopatología, la violencia, y los daños cognitivos en personas víctimas de la violencia. Se sistematizaron 50 artículos en total de las bases de datos PubMed, ScienceDirect, PsicoDoc, Proquest, y Google Académico. Las palabras claves fueron: salud mental*violencia, psicopatología*violencia, daño cognitivo*violencia, mental health*violence, cognitive imparment ${ }^{*}$ violence, war*mental health, traumatic stres*brain injury, entre los años 2008-2018. Los resultados indican que los daños en salud mental más frecuentes derivados de actos violentos son el trastorno por estrés postraumático (TEPT) que genera daños cognitivos en atención, memoria de trabajo, y aprendizaje. Estas son funciones cognitivas necesarias para un desempeño óptimo de la vida diaria. Además el TEPT desencadena depresión, ansiedad, y otras alteraciones psiquiátricas como el trastorno afectivo bipolar, o la esquizofrenia.

Palabras Clave: violencia, estrés postraumático, daño cerebral, salud mental

Artículo de investigación. Citar como: Herrera, M. E. J. y Cañas Betancur, D.C. (2020). El estrés postraumático como precursor de daños en salud mental y cognición en víctimas de violencia. Diversitas: Perspectivas en Psicología, 16(2), 311-323. https://doi.org/10.15332/22563067.6297

Autor de correspondencia: Edna Johanna Herrera Merchán, Ph. D. en Neuropsicología Clínica. Facultad de Investigaciones Universidad Manuela Beltrán. Vinculada al Grupo de Investigación en Ciencias Sociales, Humanidades y Educación CISNHE, Universidad Manuela Beltrán (Bucaramanga, Colombia). Dirección: Calle 33 No 27-12. PBX: 6525202. Correo electrónico institucional: edna.herrera@docentes.umb.edu.co.

Magister en psicología con énfasis en cognición y desarrollo moral, Faculta de Psicología, Universidad Cooperativa de Colombia. Vinculada al Grupo de Investigaciones Familia y Sociedad. Universidad Cooperativa de Colombia (Bucaramanga, Colombia). Dirección: Carrera 31 con calle 32. PBX: 6854500 . Correo electrónico institucional: dora.canas@campusucc.edu.co. 


\section{Abstract}

This article aims to demonstrate how violence is a socially damaging act that generates mental health detriment and cognitive impairment. A systematic review of articles was carried out based on the PRISMA guidelines. Studies found were related to psychopathology, violence, and cognitive impairment in victims of violence. A total of 50 articles, dated from 2008 to 2018, were systematized in databases such as PubMed, ScienceDirect, PsicoDoc, Proquest, and Google Scholar. The keywords were health*violence, psychopathology*violence, cognitive impairment*violence, mental health*violence, cognitive impairment*violence, war*mental health, traumatic stress*brain injury. The results indicate that the most frequent mental health damage derived from violent acts is the post-traumatic stress disorder (PTSD) that causes cognitive impairment in attention, working memory, and learning, which are necessary cognitive functions for optimal performance in daily life. Besides, PTSD triggers disorders such as depression, anxiety, and other psychiatric diseases such as bipolar affective disorder or schizophrenia.

Keywords: violence, post-traumatic stress disorder, brain damage, mental health.

\section{Introducción}

La salud mental debe ser concebida como "un estado de bienestar en el cual el individuo es consciente de sus propias capacidades, puede afrontar las tensiones normales de la vida, puede trabajar de forma productiva y fructífera y es capaz de hacer una contribución a su comunidad" Organización Mundial de la Salud (oms, 2013, s. p.), y no solamente vista desde la ausencia de enfermedad. Además, los individuos deben tener la capacidad de afrontar tensiones en la vida cotidiana con características de salud mental normal que generalmente no se encuentran en las personas víctimas de violencia, debido a que estas secuelas pueden vincularse con el estrés postraumático (Rodríguez de Armenta, 2007). Entendido el estrés como una condición circunstancial en la que se puede afrontar o no, dependiendo de los recursos personales (e. g., manejo emocional, conocimiento del problema y estrategias claves como para su afrontamiento), esta situación de estrés genera respuestas fisiológicas particulares como: aumento en la sudoración, aumento de palpitaciones, nudo en la garganta, sentimiento de angustia, sudoración excesiva al tener que afrontar la situación que dispara dicho estado (Amaris Macías, Madariaga, Valle y Zambrano, 2013).

Se ha reportado que mantener esta respuesta al estrés produce cambios estructurales y funcionales en el sistema nervioso. Debido a este impacto si la situación estresora se puede resolver o se aprende a afrontarla, la persona recupera un estado de bienestar y de respuestas fisiológicas diferentes a las reportadas ante el estrés, lo que indica que el sistema nervioso se adapta para el manejo de dicha condición y podrá afrontarse de una forma adecuada manteniendo una adecuada salud mental y física (Amarís Macías, Madariaga, Valle, Zambrano, 2013). Pero un nivel elevado de estrés agudo o crónico de tipo traumático generará cambios a nivel fisiológico que predispondrán al desarrollo de enfermedades y trastornos en la salud mental (Heyman et al., 2018; Liy et al., 2017; Seijas-Gómez, 2013).

Una situación que se reporta podría generar estrés traumático es la violencia, definida como "cualquier acto de comisión u omisión y cualquier condición que resulte de dichos actos, que prive a los sujetos de igualdad de derechos y libertades e interfiera con su máximo desarrollo y libertad de elegir" (Espín Falcón, Valladares González, Abad Araujo, Presno Labrador y Gener Arencibia, 2008, p. 3) y que produce cambios drásticos en las personas no sólo a nivel psicológico debido a un acto violento, sino por los cambios cerebrales/fisiológicos que se experimentan y que inciden en problemas de salud mental y deterioro cognitivo.

El objetivo de este estudio fue desarrollar una revisión sistemática con la guía PRISMA para analizar los efectos que produce la violencia en la salud mental y áreas cognitivas de personas víctimas, también en la revisión se busca conocer la relación del daño en 
salud mental de personas expuestas a condiciones de violencia y como dichas afectaciones impiden un desarrollo cotidiano en las actividades de forma normal.

\section{Metodología}

Se revisó la información relacionada con la psicopatología, la violencia, y los daños cognitivos en personas víctimas de la violencia. Luego, se sistematizaron los estudios evaluados para determinar si identificaban el objetivo en común del estudio, es decir, describir las consecuencias que genera la violencia en la salud mental y sus características, además de observar cómo la violencia también está relacionada con el estrés postraumático y cómo este incide en las alteraciones cognitivas.

Para desarrollar esta investigación sistemática se utilizó la guía para revisiones sistemáticas y metanálisis PRISMA (Cardona, Higuita y Rios, 2015). Brevemente, la estrategia consistió en definir un filtro de búsqueda de las fuentes de información a utilizar, realizando la elección de palabras clave en bases de datos como PubMed, ScienceDirect, PsicoDoc, Proquest, y google académico. Las palabras clave utilizadas fueron: "salud mental*violencia”, "psicopatología*violencia”, "daño cognitivo*violencia", "mental health*violence", "cognitive imparment*violence", "war*mental health", "traumatic stres*brain injury", "postraumatic disorders*violence*mental health", "Mental Health*war*cognitive imparment", "cognitive imparment"postraumatic disorder*violence", "postraumatic disorder*violence*brain". Se analizaron estudios desde el 2008 hasta el 2020 para un total 41 estudios, pero se tuvieron en cuenta estudios de alto impacto que pudieran describir daños en salud mental de personas víctimas de violencia de años anteriores al 2008, para lo cual se incluyeron 10 estudios más.

La información extraída de los artículos encontrados fue: el país, el tipo de estudio, valoraciones psicológicas o psiquiátricas aplicadas a sujetos, estrés postraumático en víctimas/daño en salud mental, daños cognitivos por estrés postraumático, tipo de análisis, conclusiones y resumen. Posteriormente a este análisis de revisión de los artículos aplicando los criterios de inclusión y exclusión se seleccionó un total de 50 artículos que contenían las categorías estudiadas, esta revisión con el flujo total de artículos se encuentra en la Figura 1 y la Tabla 1.

\section{Resultados}

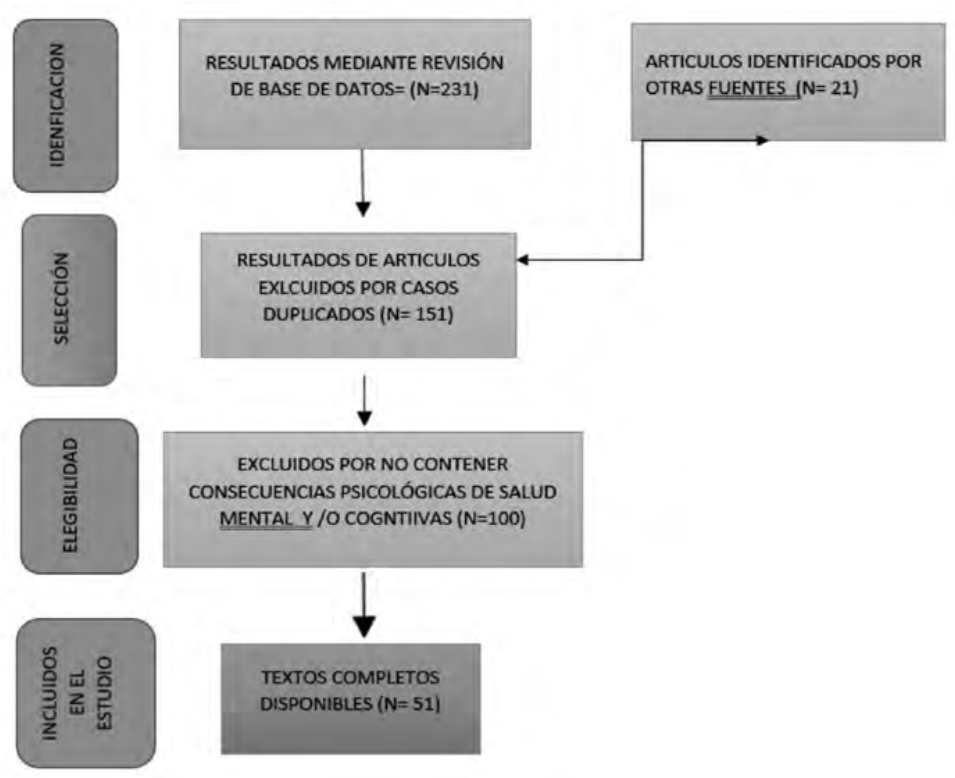

Figura 1. Diagrama de flujo representando la selección de los artículos

Fuente: elaboración propia. 
Tabla 1.

Resultados de los artículos utilizados para el presente revisión sistemática.

\begin{tabular}{lcc}
\hline \multicolumn{1}{c}{ Dimensiones } & Indicadores & Autores \\
\hline Estrés postraumati- & -Eventos violentos & -Desarrollo de trastorno de \\
co, daño fisiológico & $\begin{array}{c}\text { estrés postraumático (con- } \\
\text { secuencias fisiológicas). }\end{array}$ & Kobayashi, I., Lavela J., Bell K. y Mellman, T. A. (2016)
\end{tabular}

Barreto, L., Dimenstein, M. y Ferreira-Leite, J. (2013)

García, M. J. y Matud, M. P. (2015)

Dallos, M., Pinzon, A., Barrera, C., Mujica, J. y Meneses, Y. (2008)

-Problemas en salud men- Heyman, R., Kogan, C., Foran, H., Burns, S. ... Reed, G. (2018)

Salud mental y violencia

Conceptos sobre salud mental, estrés psicológico, estrés postraumático

Daños cerebrales asociados a estructuras cerebrales en personas víctimas de violencia

Salud pública, salud mental y violencia
Conceptos básicos sobre estrés postraumático.

- Vivencias traumáticas y desarrollo del TEPT

- Daños específicos en áreas cerebrales como hipocampo, amígdala, sistema límbico, lóbulos frontales y prefrontales asociados a TEPT.

- Daños en áreas cerebrales asociados a la violencia.

Salud pública y estrés postraumático Violencia
Hidalgo-Ruzzante, N., Gómez, P., Buesolzquierdo, N., Jiménez, P., Martín, E. y Pérez-García, M. (2012)

Imanza-Avendaño, A. M., Gómez-San, L. A. H. y Gurrola-Peña, G. M. (2018)

Merino, V. (2015)

Montenegro, A. (2018)

Rodríguez de Armenta, M.J. (2007).

Herrera, Cañas, González, Uribe y Padilla (2020)

Organización Mundial de la Salud (2013).

Macías, M. A., Madariaga Orozco, C., Valle Amarís, M., y Zam-

brano, J. (2011)

Aupperle, R. L., Melrose, A. J., Stein, M. B y Paulus, M. P. (2012)

Belmonte, C. (2007)

Blair, K. S., Vythilingam, M., Crowe, S. L., McCaffrey, D. E., Ng, P., ... Blair, R. J. (2013)

Bonne, O., Vythilingam, M., Inagaki, M., Wood, S., Neumeister, A., ... Charney, D. S. (2008)

Dajas, F. (2010)

Daniels, J. K., Frewen, P., McKinnon, M. C. y Lanius, R. A. (2011)

Eckart, C., Stoppel, C., Kaufmann, J., Tempelmann, C., Hinri-

chs, H., ... Kolassa, I. (2011)

Koenigs, M. y Grafman, J. (2009).

Li, Y., Hou, X., Wei, D., Du, X., Zhang, Q. ... Qiu, J. (2017)

Milad, M. R., Pitman, R. K., Ellis, C. B., Gold, A. L., Shin, L. M., ...Rauch, S. L. (2009)

Morey, R. A., Dolcos, F., Petty, C. M., Cooper, D. A., Hayes, J. P., ... McCarthy, G. (2009)

Morey, R. A., Gold, A. L., LaBar, K. S., Beall, S. K., Brown, V.M., ... Mid-Atlantic MIRECC Workgroup (2012)

Otte, C., Lenoci, M., Metzler, T., Yehuda, R., Marmar, C. R. y Neylan, T. C. (2005)

Schweizer, S. y Dalgleish, T. (2011)

White, S. F., Costanzo, M. E., Blair, J. R., Roy, M. J. (2014)

Espín Falcón, J. C., Valladares González, A. M., Abad Araujo, J. C., Presno Labrador, C., y Gener Arencibia, N. (2008)

Palacios, L. y Heinze, G. (2002) 


\begin{tabular}{|c|c|c|}
\hline Dimensiones & Indicadores & Autores \\
\hline $\begin{array}{l}\text { Desplazamiento } \\
\text { forzado violencia y } \\
\text { salud mental }\end{array}$ & $\begin{array}{c}\text { Situaciones de } \\
\text { desplazamiento } \\
\text { Origen de violencias } \\
\text { sociales } \\
\text { Salud mental }\end{array}$ & $\begin{array}{l}\text { Alejo E. G. (2005) Alejo, E., Rueda, G., Ortega, M. y Orozco, L. } \\
\text { (2007) } \\
\text { Andrade-Salazar, J. A. (2008) } \\
\text { Andrade, J., Parra, M. y Torres, D. (2013) } \\
\text { Bell, V., Méndez, F., Martínez, C., Palma, P. P. y Bosch, M. } \\
\text { (2012) } \\
\text { Campo-Arias, A., Oviedo H. y Herazo, E. (2014) } \\
\text { Hewitt Ramírez, N., Juárez, F., Parada Baños, A. J., Guerre- } \\
\text { ro Luzardo, J., Romero Chávez, Y. M., ... Vargas Amaya, M. V. } \\
\text { (2016) } \\
\text { Palacio, C.A. (2016). } \\
\text { Defensoría del Pueblo. (2012). } \\
\text { Chavez, M., Petrzelov, J., Zapata Salazar, J., Hern, M., y Rodr, } \\
\text { M. (2015) } \\
\text { Londoño, N., Patiño, C., Restrepo, D., Correa, J., Raigoza, J., ... } \\
\text { Rojas, C. (2008) } \\
\text { Jaimes (2014) }\end{array}$ \\
\hline $\begin{array}{l}\text { Consecuencias del } \\
\text { estrés postraumá- } \\
\text { tico y desordenes } \\
\text { del comportamien- } \\
\text { to (psicopatología) }\end{array}$ & $\begin{array}{l}\text { Psicopatología relacionada } \\
\text { por estrés postraumático } \\
\text { con origen en la violencia }\end{array}$ & $\begin{array}{l}\text { Gómez-Restrepo, C., Tamayo-Martínez, N., Buitrago, G., Guarni- } \\
\text { zo-Herreño, C., Garzón-Orjuela, N., .. Rincón, C. (2016) } \\
\text { González, J. y Pardo, E. (2007). } \\
\text { Labrador, F., Fernández-Velasco, R. y Rincón, P. (2010). } \\
\text { Paz, P., Labrador, F. y Crespo, M. (2004) } \\
\text { Ribeiro, W. S., Andreoli, S. B., Ferri, C. P Prince, M. y Mari, J. } \\
\text { J. (2009) } \\
\text { Sarasua, B., Zubizarreta, I., Echeburúa, E. y Corral, P. (2007) } \\
\text { Tamayo, N., Rincón, C. J., de Santacruz, C., Bautista, N., Colla- } \\
\text { zos, J., Gómez-Restrepo, C. (2016) } \\
\text { Vieyra Moreno C. I., Gurrola Peña G. M., Balcazar Nava P., Boni- } \\
\text { lla Muñoz M. P. y Virseda Heras, J. A. (2009) } \\
\text { Ayala, R.; De Paúl Ochotorena, J. (2004) }\end{array}$ \\
\hline
\end{tabular}

Fuente: elaboración propia a partir de Cardona y otros (2015)

\section{Estrés Postraumático y Violencia en Colombia}

De acuerdo con un análisis desarrollado por Alejo (2005) expone que los desplazamientos por hechos violentos generan estilos de vida desadaptados que producen una dificultad para la integración social, además establece una relación entre el estrés postraumático y la violencia en Colombia reflejada principalmente en el fenómeno del desplazamiento, teniendo en cuenta que las cifras son alarmantes para éste fenómeno "desde 1997 hasta el 2008, en Colombia se desplazaron 747.719 hogares" (Andrade-Salazar, 2008, p.115), y desde el 2003 hasta el 2012 en total se han desplazado 2'729.153 personas, un número alarmante que sólo es rebasado por países como Siria, y República Central Africana (Jaimes Villamizar, 2014). Esta violencia que genera el desplazamiento tiene orígenes socio-políticos de grupos armados en poblaciones colombianas, la guerra por la toma forzada de tierras produce desplazamiento y por ende un daño a la salud mental de sus habitantes (Andrade-Salazar, 2008).

Una gran proporción de las víctimas desplazadas han vivido un sinnúmero de situaciones violentas que generan la salida de campesinos hacía un futuro incierto (Alejo, 2005), condición que agrava el fenómeno del desplazamiento, donde muchas de éstas persona no tienen redes de apoyo y se ven forzadas a sobrevivir en grandes ciudades sin tener oportunidades laborales o educativas. Ante estas alternativas desalentadoras, muchas personas que han sufrido la violencia tienden a desarrollar trastornos mentales como lo indica el estudio de Tamayo y colaboradores (2016). En este estudio 
se encontró que la población desplazada víctima de la violencia presentaba trastornos mentales en mayor grado que la población general. Estos se evidenciaron en trastornos de ansiedad por la vida, trastornos del afecto, ideación suicida y planeación suicida, consumo de alcohol, y estrés postraumático. Lo anterior indica, que las situaciones de violencia terminan desarrollando trastornos mentales que puedan afectar el normal funcionamiento en la vida cotidiana (Chávez, Petrzelová, Zapata, Hernández y Rodríguez, 2015; Herrera Merchán, Cañas Betancur, González, Uribe y Padilla, 2020; Ribeiro, Andreoli, Ferri, Prince y Mari, 2009).

De acuerdo con Alejo (2005) el Trastorno de Estrés Postraumático (TEPT) se evidencia en los síntomas de la población víctima de violencia en Colombia, tales como el recuerdo recurrente del hecho traumático, las ilusiones (flashback) como si el evento se viviese de nuevo, evitación de personas o lugares que le recuerden el hecho, aplanamiento emocional, incapacidad para expresar emociones y aislamiento social (Alejo, 2005). Todas estas características del trastorno de estrés postraumático, según Alejo (2005) van a generar problemas de salud mental comórbidos con el TEPT como el trastorno del estado de ánimo y la depresión (Alejo, Rueda, Ortega y Orozco, 2006).

Andrade-Salazar (2008) expone un estudio donde se evaluaron 36 familias desplazadas por el conflicto Armado y reasentadas en el municipio del CairoValle, reportan que el $100 \%$ de la población evaluada presenta estrés postraumático, y depresión en una tasa de dos mujeres por cada hombre, es decir, que en género la mujer mostró más sintomatología depresiva y trastorno del estrés postraumático que el grupo masculino, lo que indica que los cuadros psicopatológicos perduran más en las mujeres a pesar de que las condiciones de seguridad puedan mejorar. También expone que la presencia de depresión en hombres reactiva un Trastorno Afectivo Bipolar tipo I (i.e., manía) que puede generar una exteriorización de la agresividad y fomentar un ambiente de violencia intrafamiliar, revictimizando a sus compañeras, lógicamente este tipo de daño a la salud mental interfiere en el desempeño laboral, en el clima laboral, y a nivel de la comunidad. En este sentido esta problemática social genera una afectación en la salud mental que repercute en el funcionamiento normal de la vida cotidiana de una persona o una comunidad (Andrade, Parra y Torres, 2013).

Por otro lado, Ayala y De Paúl Ochotorena (2004) proponen que la mayor parte de las personas que han sufrido situaciones traumáticas pueden tener un factor de riesgo para la aparición del TEPT, entendido como un trastorno de ansiedad desencadenado por un hecho traumático pero precisando que no todos los que se exponen a un hecho traumático desarrollarán el TEPT (Imanza-Avendaño, Gómez-San, y Gurrola-Peña, 2018; Montenegro, 2018). Este trastorno puede presentarse en mayor proporción en la población femenina y dando una mayor vulnerabilidad a sufrir violencia sexual u otro tipo de violencia por ser mujeres (Sarasua, Zubizarreta, Echeburúa y Corral, 2007). Otra característica que lo define es su subdiagnóstico, y es posible que aumente el número de personas con TEPT debido a los hechos violentos que se sufren en las sociedades como la colombiana, vulnerables tanto económica como socialmente. A este subdiagnóstico se suma la falta de un tratamiento adecuado como sí lo reciben otros trastornos psiquiátricos (e.g., la esquizofrenia, o el trastorno afectivo bipolar) esto debido a que se conceptualiza el TEPT como el desarrollo sintomático normal de haber sido expuesto a un evento traumático (Palacios y Heinze, 2002).

Por otro lado, la edad es un factor importante para el desarrollo del TEPT, así, los individuos más jóvenes que experimentan hechos traumáticos presentan una mayor probabilidad de desarrollar el TEPT (Palacios y Heinze, 2002). Ayala y De Paúl Ochotorena (2004), reportan que personas víctimas de violación, inundaciones con percepción de muerte o con heridas graves son más vulnerables para desarrollar un TEPT, al igual que víctimas de violencia sexual casi todas desarrollaron TEPT, otros factores fueron "haber sido aterrorizadas o haber tenido miedo de uno de los padres (o padrastro que la haya amenazado) que tenía reacciones impredecibles a causa del abuso de alcohol o drogas" (Martín y De Paúl, 2004, p. 47). Es decir, haber sido víctima de malos tratos en la infancia es un factor determinante para desarrollar TEPT. Las personas que desarrollaron TEPT mostraron mayores problemas de 
depresión y ansiedad, hecho que se confirma con otros autores (Bell, Méndez, Martínez, Palma, y Bosch, 2012; Defensoría del Pueblo, 2012; Hewitt Ramírez et al., 2016; Labrador, Fernández-Velasco y Rincón, 2010; Ayala y De Paul, 2004).

En la investigación de Sarasua y colaboradores (2007), se encontró que en una muestra de 148 mujeres, aquellas de edades más jóvenes (18 a 29 años) tendían a mostrar mayores problemas de TEPT que mujeres mayores (>30 años). También se reportó que las mujeres más jóvenes habían sufrido maltrato físico más significativo que las mujeres mayores en un $71 \%$, y la amenaza con arma se presentó en mayor proporción en mujeres jóvenes que en mayores ( $48 \%$ ), lo que muestra que han visto amenazada su integridad física, de acuerdo con esto, las víctimas jóvenes presentaron mayores puntajes en depresión, y un grado de autoestima más bajo. Las mujeres que presentaron TEPT mostraron mayores niveles de malestar emocional que aquellas que no lo presentaban, con el agravante de mostrar altos niveles de ansiedad, depresión, un grado de valoración negativa acerca de sí mismas y mayor inadaptación en sus vidas cotidianas (García y Matud, 2015; Sarasua et al., 2007).

En el estudio de Londoño y colaboradores (2008) encontraron que en víctimas de la violencia del municipio de Bojayá se presentaron problemas de resiliencia especialmente en aquellos que presentaban TEPT mostrando características en el perfil cognitivo como:

[...] baja autoestima, distanciamiento afectivo hacia otros y la religión, inhibición para expresar dificultades emocionales relacionadas con el afrontamiento al estrés, y búsqueda del recurso profesional. En tanto quienes no desarrollaron TEPT mostraron mejor resiliencia caracterizado por sobrevaloración de sí mismos, acercamiento a la religión y expresión de sus necesidades emocionales (Londoño et al., 2008 p. 23),

Lo reportado en Londoño y colaboradores (2008) muestra como el estrés postraumático afecta procesos de resiliencia, autoestima (Barreto, Dimenstein y Ferreira-Leite, 2013), y afrontamiento al estrés, por estos motivos es necesario intervenir en las manifestaciones tempranas del estrés postraumático intentando favorecer otros procesos psicológicos que ayuden a la salud mental.

Según González y Pardo (2007) los pacientes con TEPT en su componente emocional se pueden encontrar fenómenos de irrealidad de que la situación traumática de violencia no ocurrió, y esta sensación puede venir acompañada de llanto intenso, de síntomas psiquiátricos como confusión, desorientación y problemas para procesar la información debido al hecho traumático. Adicionalmente, se presentan sentimientos de indefensión, y problemas en la toma de decisiones, todo esto puede ser la antesala a la aparición de trastornos de adaptación, ansiedad, depresión, y el estrés postraumático.

Esto sugiere que las personas que han sufrido eventos traumáticos donde han visto vulnerada su integridad física desarrollan TEPT, generando comorbilidad con trastornos de ansiedad y depresión, relacionados con baja autoestima, baja resiliencia y problemas para adaptarse y desarrollar una vida normal.

\section{Estrés Postraumático y Daños en la Salud Mental}

Las alteraciones más frecuentes de salud mental debidas a hechos violentos son el desarrollo del TEPT, depresión y ansiedad, especialmente encontrado en personas víctimas del conflicto armado (Bell et al., 2012; Defensoría del Pueblo, 2012; Hewitt et al., 2016). En este sentido, Eckart y colaboradores (2011) reportaron que en personas que habían desarrollado estrés postrauma en comparación con controles se detectó que en un grupo de 20 personas con TEPT, 15 desarrollaron depresión con un mayor grado de suicidio en comparación con muestras controles. Por otro lado, los trastornos del sueño son una característica típica de personas con TEPT, representados en pesadillas, insomnio; que se caracteriza por la dificultad para la conciliación del sueño, o despertares continuos (Kobayashi, Lavela,Bell y Mellman 2016; Otte et al., 2005).

Existe evidencia que sugiere la relación entre el TEPT en personas víctimas de la violencia por 
desplazamiento en Colombia y su relación con la aparición de altos niveles de problemas de salud mental en dicha población (Alejo, 2005; Bell et al., 2012; Campo-Arias, Oviedo y Herazo, 2014; Defensoría del Pueblo, 2012; Gómez-Restrepo et al., 2016; Hewit et al., 2016; Londoño et al., 2008; Tamayo et al., 2016).

Así mismo, Vieyra y colaboradores (2009) encontraron en una muestra de 30 mujeres víctimas de violencia síntomas de salud mental alterados en características como la somatización, la sensibilidad personal, el psicoticismo y la depresión. Hallaron que las mujeres que habían sufrido violencia solían presentar síntomas físicos como consecuencia de una afectación en la salud mental. Esto genera una peor percepción de calidad de vida en dichas mujeres, y una mayor utilización de servicios de salud. Vieyra y colaboradores (2009) definen la somatización como la percepción pobre de bienestar, manifestando sentir dolor muscular o malestares gastrointestinales, y la alteración de comportamientos sociales, familiares e interpersonales; así, esta variable de somatización fue la más afectada. En cuanto al psicoticismo que hace referencia a la conmoción, aturdimiento, desorientación y sentimientos de soledad que experimenta la víctima de violencia, con cambios drásticos y nocivos en aspectos emocionales como suele ser "el paso del miedo a la rabia hacia el agresor, de la compasión hacia sí misma a la culpa, y de la tristeza a la euforia" (Vieyra et al., 2009, p. 63) también fue reportada. De igual forma, en esta muestra de mujeres se encontró que la violencia, siendo un factor social, altera funciones de salud física y mental de vital importancia como son la resiliencia, la autoestima, y facilita la aparición de síndromes ansiosodepresivos, en definitiva, el estrés postraumático es la antesala a daños en la salud mental, y por ende es un daño a la salud pública (Bell et al., 2012; Defensoría del Pueblo, 2012; Hewitt et al., 2016; Vieyra et al., 2009).

En resumen, los actos violentos podrían aumentar la probabilidad en la aparición de trastornos mentales en sus víctimas, entre las más mencionadas se encuentran el Trastorno de Estrés Postraumático, factor comórbido que aumentará la probabilidad de depresión y trastornos de ansiedad (Dallos et al., 2008; Paz, Labrador y Crespo, 2004), y rasgos psicopatológicos entre los que se encuentran paranoia, rasgos psicosomáticos, síntomas psicóticos similares a la esquizofrenia (Andrade et al., 2013; Merino, 2015; Vieyra et al., 2009). Finalmente, es necesario determinar cuáles son los cambios neurofisiológicos que determinan el daño cerebral que produce la exposición repetida a actos violentos que se deriva en un TEPT.

\section{Estrés Postraumático y Daños Cognitivos}

Factores estresores pueden generar anomalías tanto funcionales como estructurales en varias áreas cerebrales tales como el hipocampo que se asocia con la memoria episódica y autobiográfica, y que con alteraciones hipocampales dichos recuerdos pueden ser confusos o distorsionados (Li et al., 2017). Así mismo, los núcleos amigdalinos, que se relacionan con la generación de alarmas en situaciones de peligro, encargados también de generar procesos de empatía emocional, y permiten la supervivencia humana, cuando existe un TEPT se reporta una hipersensibilidad, generando respuestas exageradas de miedo, un alto nivel de excitación emocional y una respuesta hiperreactiva de los núcleos de la amígdala (White, Costanzo, Blair y Roy, 2014). Así, esta estructura ha sido relacionada con trastornos de ansiedad (Li et al., 2017) y una disminución en el volumen de la amígdala izquierda y derecha se asocia con TEPT (Morey et al., 2012).

Cuando existe un estrés traumático, el aumento de los niveles de glucocorticoides mantienen el estado de alerta del cuerpo, debido a que se segregan altos niveles de cortisol que van a generar en el organismo un alto nivel de estrés y por ende el cuerpo empieza a enfermar (Seijas-Gómez, 2013). Los altos niveles de esta neurohormona también se han relacionados con la disminución del tamaño del hipocampo. Así, los problemas de memoria están asociados también a una reducción del hipocampo posterior en personas que han sufrido TEPT (Bonne et al., 2008; Morey et al., 2012), por tal motivo, una disminución del mismo podrá incidir en procesos de aprendizaje y disminución de los niveles cognitivos (Dajas, 2010; Seijas-Gómez, 2013). 
Esta afirmación se puede evidenciar en los estudios de Daniels y colaboradores (2011), en donde proponen un modelo de afectación cerebral en personas que han sufrido episodios de violencia. Como se describió previamente, existe un daño en el eje hipotálamo-hipofisiario debido a los continuos episodios de violencia que generará en la persona un estado de hipervigilancia (Seijas-Gómez, 2013). Esta activación afectará el sistema atencional y por ende ante estímulos neutros o no aversivos se producirá una sobreexcitación emocional, que es un síntoma característico de persona con problemas de estrés postraumático (Schweizer y Dalgleish, 2011).

A nivel neuronal pueden existir problemas de aprendizaje debido a un estrés prolongado y se ven alteradas la memoria episódica, el procesamiento emocional, el control ejecutivo, y las alteraciones asociadas de memoria (Seijas-Gómez, 2012; Eckart y otros, 2011). De la misma forma, se genera una falta de control regulativo de respuestas emocionales ante estímulos neutros relacionado con hiperactividad de la amígdala cerebral (White et al., 2014; Blair et al., 2013). Se han encontrado problemas de atención debido a una hiperreactividad de la amígdala a estímulos externos y áreas cerebrales encargadas de éstos procesos que no permiten mantener niveles de concentración cognitiva adecuados, asociados a trastornos de ansiedad derivados de una sobre-excitación de la amígdala (White et al., 2014; Milad et al., 2009; Koenigs y Grafman, 2009), esto se puede demostrar en estudios que han encontrado cortisol en saliva de mujeres que han sido víctimas de la violencia (Hidalgo-Ruzzante et al., 2012).

Los autores concluyen que una disminución del hipocampo posterior en una persona con TEPT estará asociado a una deficiencia en los mecanismos adaptativos para el manejo del estrés, es decir, una incapacidad para afrontar el estrés a comparación de una persona sin disminución de hipocampo posterior, quien podría adaptarse a las nuevas situaciones y manejar un estrés cotidiano (Bonnes et al., 2008), además de problemas en memoria de trabajo para el almacenamiento de la información compleja y las operaciones cognitivas (Aupperle, Melrose, Stein y Paulus, 2012; Milad et al., 2009; Morey et al., 2009; Schweizer y Dalgleish, 2011).

\section{Discusión y Conclusiones}

Los actos violentos generan consecuencias graves en la salud mental de la población (Hewitt Ramírez et al., 2016; Li et al., 2017; Seijas-Gómez, 2013;), varios estudios muestran que los efectos nocivos que genera la violencia en el sistema nervioso están asociados a una disminución del crecimiento celular de estructuras como el hipocampo (Bonne et al., 2008; Morey et al., 2012) y generando hiperreactivación de la amígdala (Blair et al., 2013; White et al., 2014) además de disminución del volumen de la sustancia gris a nivel cerebral (Daniels et al., 2011). Todos estos daños neurológicos van a incidir en la salud mental de la población, especialmente cuando se desarrolla el trastorno de estrés postraumático que es catalogado como un trastorno de ansiedad de características nocivas como evitación de lugares y personas que evocan el episodio traumático, reviviscencia de memorias traumáticas (flashbacks), problemas para la conciliación del sueño y constantes pesadillas referidas al hecho traumático (Alejo, 2005; Otte et al., 2005).

Finalmente, el TEPT es el inicio de otros trastornos psiquiátricos posteriores, tales como la depresión, los trastornos afectivos bipolares, y la psicosis con síntomas similares a la esquizofrenia (Alejo et al., 2006; Eckart et al., 2011; Hewitt Ramírez et al., 2016). Todo esto no permite que existan factores favorables a nivel psicológico como la resiliencia, la adecuada autoestima, la capacidad de afrontamiento y por ende la adaptación (Londoño et al., 2008). En definitiva, el cerebro después de un evento traumático repetitivo tras la generación de glucorticoides empezará a generar un daño celular (Seijas-Gómez, 2013) en estructuras que permiten la comprensión e interpretación emocional de la realidad determinando la supervivencia y por ende a la adaptación social, estas estructuras son, el hipocampo y la amígdala cerebral (Belmonte, 2007), además de otras estructuras que generan un daño cerebral y por ende se convierte en una amenaza para el desarrollo de trastornos psiquiátricos iniciando con el TEPT (Li et al., 2017). Motivo por el cual una persona víctima de violencia, tendrá grandes repercusiones en su salud mental, y en áreas cognitivas que entorpecerá un normal desarrollo de la vida cotidiana. 


\section{Referencias}

Alejo E. G. (2005). Aplicación del modelo de estrés postraumático en el estudio del impacto de la violencia sobre la salud mental en población desplazada. MedUNAB, 8(1), 23-28.

Alejo, E., Rueda, G., Ortega, M. y Orozco, L. (2007). Estudio epidemiológico del trastorno por estrés postraumático en población desplazada por la violencia política en Colombia. Universitas Psychologica, 6 (3), 623-635.

Amarís Macías, M., Madariaga, C., Valle, M. y Zambrano, J. (2013). Estrategias de afrontamiento individual y familiar frente a situaciones de estrés psicológico Individual. Psicología desde el Caribe, 30(1), 123-145.

Andrade-Salazar, J. A. (2008). Efectos psicopatológicos del conflicto armado Colombiano en familias en situación de desplazamiento forzado reasentadas en el municipio del Cairo en el año 2008. Revista Científica Ciencias Humanas, “Orbis" 7(20), 111-114.

Andrade, J., Parra, M. y Torres, D. (2013). Desplazamiento forzado y vulnerabilidad en la salud mental en Colombia. Revisión del Estado del arte. Revista Electrónica de Psicología Social «Poiésis», 0(25), 1-16.

Aupperle, R. L., Melrose, A. J., Stein, M. B y Paulus, M. P. (2012). Executive function and PTSD: Disengaging from trauma. Neuropharmacology, 62(2), 686-694. http://doi.org/10.1016/j. neuropharm.2011.02.008.

Ayala, R. y De Paúl Ochotorena, J. (2004). Trastorno por estrés postraumático en víctimas de situaciones traumáticas. Psicothema, 16(1), 45-49.

Barreto, L., Dimenstein, M. y Ferreira-Leite, J. (2013). Atenção a mulheres em situação de violência com demandas em saúde mental. Athenea Digital, 13(3), 195-207.

Bell, V., Méndez, F., Martínez, C., Palma, P. P. $y$ Bosch, M. (2012). Characteristics of the
Colombian armed conflict and the mental health of civilians living in active conflict zones. Journal Conflict and Health, 6(1), 1-8. http://doi.org/10.1186/1752-1505-6-10.

Belmonte, C. (2007). Emociones y Cerebro. Revista de la Real Academia de Ciencias Exactas, Fisicas y Naturales, 1(101), 59-68.

Blair, K. S., Vythilingam, M., Crowe, S. L., McCaffrey, D. E., Ng, P., ... Blair, R. J. (2013). Cognitive control of attention is differentially affected in trauma-exposed individuals with and without post-traumatic stress disorder. Psychology Medicine, 43(1), 85-95. https://doi.org/10.1017/ S0033291712000840

Bonne, O., Vythilingam, M., Inagaki, M., Wood, S., Neumeister, A., ... Charney, D. S. (2008). Reduced Posterior Hippocampal Volume in Posttraumatic Stress Disorder. Journal of Clinical Psychiatry, 69(7), 1087-1091.

Cardona-Arias, J. A., Higuita-Gutiérrez, L. F. y Ríos Osorio, L. A. (2016). Revisiones sistemáticas de la literatura científica: la investigación teórica como principio para el desarrollo de la ciencia básica y aplicada. Bogotá: Ediciones Universidad Cooperativa de Colombia. http:// dx.doi.org/10.16925/9789587600377

Campo-Arias, A., Oviedo H. y Herazo, E. (2014). Prevalencia de síntomas, posibles casos y trastornos mentales en víctimas del conflicto armado interno en situación de desplazamiento en Colombia: una revisión sistemática. Revista Colombiana de Psiquiatría, 43(4), 177-185.

Chávez, M., Petrzelová, J., Zapata, J., Hernández, M. y Rodríguez, M. (2015). Violencia psicosocial en el noreste de México: repercusiones en la salud mental. Psicogente, 18(33), 11-21. http:// doi.org/10.17081/psico.18.33.52

Dallos, M., Pinzon, A., Barrera, C., Mujica, J. y Meneses, Y. (2008). Impacto de la violencia sexual en la salud mental de las victimas en Bucaramanga, Colombia. Revista Colombiana de Psiquiatría, 37(1), 58-63. 
Dajas, F. (2010). El Cerebro Violento. Sobre la psicobiología de la violencia y los comportamientos agresivos. Revista de Psiquiatría del Uruguay, 74(1),22-37.

Daniels, J. K., Frewen, P., McKinnon, M. C. y Lanius, R. A. (2011). Default mode alterations in PostTraumatic Stress Disorder related to early-life trauma: a developmental perspective. Journal of Psychiatry and Neuroscience, 36(1), 56-59.

Defensoría del Pueblo. (2012). Protocolo de orientación y atención a víctimas de tortura y otros tratos crueles, inhumanos y degradantes en el marco del conflicto armado interno, por la delegada para la orientación y asesoría a las víctimas del conflicto armado interno. Recuperado de http://www.defensoria.org.co/red/anexos/ pdf/04/pt_tortura

Eckart, C., Stoppel, C., Kaufmann, J., Tempelmann, C., Hinrichs, H., ... Kolassa, I. (2011). Structural alterations in lateral prefrontal, parietal and posterior midline regions of men with chronic posttraumatic stress disorder. Journal of Psychiatry and Neuroscience, 36(3), 176-186. https://dx.doi.org/10.1503/jpn.100010

Espín Falcón, J. C., Valladares González, A. M., Abad Araujo, J. C., Presno Labrador, C., y Gener Arencibia, N. (2008). La violencia, un problema de salud. Revista Cubana de Medicina General Integral, 24(4).

García, M. J. y Matud, M. P. (2015). Salud mental en mujeres maltratadas por su pareja. Un estudio con muestras de México y España. Salud Mental, 38(5), 321-327. https://dx.doi. org/10.17711/SM.0185-3325.2015.044.

Gómez-Restrepo, C., Tamayo-Martínez, N., Buitrago, G., Guarnizo-Herreño, C., Garzón-Orjuela, N., ... Rincón, C. (2016). Violencia por conflicto armado y prevalencias de trastornos del afecto, ansiedad y problemas mentales en la población adulta colombiana. Revista Colombiana de Psiquiatría, 45(1), 147-153.

González, J. y Pardo, E. (2007). El daño psíquico en las víctimas de agresión sexual. Artículo publicado en el VIII Congreso Virtual de Psiquiatría (Interpsiquis 2.007.) Consultado el 10 de octubre de 2016 en http://www.psiquiatria.com/articulos/ psiquiatria_legal/29258/.

Herrera Merchán E. J., Cañas Betancur D. C., González E. C., Uribe C. y Padilla L. A. (2020). Caracterización psicosocial en una muestra de mujeres víctimas de violencia de género en Colombia: un estudio piloto. I+D Revista de Investigaciones, 15(2), 125-136.

Heyman, R., Kogan, C., Foran, H., Burns, S. ... Reed, G. (2018) A case-controlled field study evaluating ICD-11 proposals for relational problems and intimate partner violence. International Journal of Clinical and Health Psychology, 18(2), 113-123.

Hewitt Ramírez, N., Juárez, F., Parada Baños, A. J., Guerrero Luzardo, J., Romero Chávez, Y. M., ... Vargas Amaya, M. V. (2016). Afectaciones psicológicas, estrategias de afrontamiento y niveles de resiliencia de adultos expuestos al conflicto armado en Colombia. Revista Colombiana de Psicología, 25(1), 125-140. https://dx.doi. org/10.15446/rcp.v25n1.49966

Hidalgo-Ruzzante, N., Gómez, P., Buesolzquierdo, N., Jiménez, P., Martín, E. y Pérez-García, M. (2012). Secuelas cognitivas en mujeres víctimas de violencia de género. 3er Congreso para el estudio de la violencia contra las mujeres.

Imanza-Avendaño, A. M., Gómez-San, L. A. H. y Gurrola-Peña, G. M. (2018). Victimización, resiliencia y salud mental de estudiantes de universidad en Tamaulipas, México. Revista Latinoamericana de Ciencias Sociales, Niñez y Juventud, 16(1), 345-360. HTTPS://DX.DOI. ORG/10.11600/1692715x.16121

Jaimes Villamizar J.P. (2014). Desplazamiento Forzado y Derechos Humanos (Tesis Doctoral). Universidad de Granada, Granada. Disponible en: https://digibug.ugr.es/bitstream/handle/10481/34695/24308961. pdf?sequence $=1$ \&isAllowed $=y$

Kobayashi, I., Lavela J., Bell K. y Mellman, T. A. (2016). The impact of posttraumatic stress 
disorder versus resilience on nocturnal autonomic nervous system activity as functions of sleep stage and time of sleep. Physiology \& Behavior, 1(164), 11-18. http://doi. org/10.1016/j.physbeh.2016.05.005.

Koenigs, M. y Grafman, J. (2009). Post-traumatic stress disorder: The role of medial prefrontal cortex and amygdala. The Neuroscientist: A Review Journal Bringing Neurobiology, Neurology and Psychiatry, 15(5), 540-548. http://doi.org/10.1177/1073858409333072

Labrador, F., Fernández-Velasco, R. y Rincón, P. (2010). Características psicopatológicas de mujeres víctimas de violencia de pareja. Psicothema, 22(1), 99-105.

Li, Y., Hou, X., Wei, D., Du, X., Zhang, Q. ... Qiu, J. (2017) Long-Term Effects of Acute Stress on the Prefrontal-Limbic System in the Healthy Adult. PLoS ONE, 12(1), e0168315. https://doi. org/10.1371/journal.pone.0168315.

Londoño, N., Patiño, C., Restrepo, D., Correa, J., Raigoza, J., ... Rojas, C. (2008). Perfil cognitivo asociado al trastorno por estrés postraumático en víctimas de violencia armada. Informes Psicológicos, 10, 11-27.

Macías, M. A., Madariaga Orozco, C., Valle Amarís, M. y Zambrano, J. (2013). Estrategias de afrontamiento individual y familiar frente a situaciones de estrés psicológico. Psicología desde el Caribe, 30(1), 123-145.

Merino, V. (2015). Las cicatrices sobre la salud mental de la violencia de género. Periódico $A B C$ de España. Recuperado de: https://www.abc. es/salud/noticias/20150331/abci-depresionviolencia-genero-201503311259.html.

Milad, M. R., Pitman, R. K., Ellis, C. B., Gold, A. L., Shin, L. M., Rauch, S. L. (2009). Neurobiological basis of failure to recall extinction memory in posttraumatic stress disorder. Biological Psychiatry, 66(12),1075-82. http://doi. org/10.1016/j.biopsych.2009.06.026
Montenegro, A. (2018). Gravedad de los síntomas del trastorno de Estrés Postraumático en mujeres víctimas de violencia física y psicológica en la Fiscalía General del Estado Unidad de Flagrancia [Tesis de pregrado]. Universidad Central del Ecuador, Quito Ecuador. Recuperado de http://www.dspace.uce.edu. ec/bitstream/25000/15417/1/T-UCE-0007PC053-2018.pdf

Morey, R. A., Dolcos, F., Petty, C. M., Cooper, D. A., Hayes, J. P., ... McCarthy, G. (2009). The role of trauma related distractors on neural systems for working memory and emotion processing in posttraumatic stress disorder. Journal of Psychiatric Research, 809-817.

Morey, R. A., Gold, A. L., LaBar, K. S., Beall, S. K., Brown, V.M., ... Mid-Atlantic MIRECC Workgroup (2012). Amygdala volume changes in posttraumatic stress disorder in a large case-controlled veterans group. Archives of General Psychiatry, 69(11), 1169-78. https://doi.org/10.1001/ archgenpsychiatry.2012.50.

Organización Mundial de la Salud (2013). Salud mental: un estado de bienestar. Recuperado de: http://www.who.int/features/factfiles/mental_health/es/ .

Otte, C., Lenoci, M., Metzler, T., Yehuda, R., Marmar, C. R. y Neylan, T. C. (2005). Hypothalamic-Pituitary- Adrenal Axis Activity and Sleep in Posttraumatic Stress Disorder. Neuropsychopharmacology, 30 (6), 1173-1180.

Palacio, C. A. (2016). La salud mental y el posconflicto. Revista Colombiana de Psiquiatría, 45(4), 229.

Palacios, L. y Heinze, G. (2002). Trastorno por estrés postraumático: una revisión del tema (primera parte). Salud Mental, 25(3), 19-26.

Paz, P., Labrador, F. y Crespo, M. (2004). Efectos psicopatológicos del maltrato doméstico. Avances en Psicología Latinoamericana, 22, 105-116. 
Ribeiro, W. S., Andreoli, S. B., Ferri, C. P Prince, M. y Mari, J. J. (2009). Exposure to violence and mental health problems in low and middleincome countries: a literature review. Revista Brasilera de Psiquiatría, 31(S2),49-57.

Rodríguez de Armenta, M.J. (2007). Violencia de género. Guía asistencial. Anuario de Psicología Jurídica, 16, 145-147.

Sarasua, B., Zubizarreta, I., Echeburúa, E. y Corral, P. (2007). Perfil psicopatológico diferencial de las víctimas de violencia de pareja en función de la edad. Psicothema, 19(3), 459-466.

Schweizer, S. y Dalgleish, T. (2011). Emotional working memory capacity in posttraumatic stress disorder (PTSD). Behavioral Research Theraphy, 49(8), 498-504.

Seijas-Gómez, R. (2013). Trastorno por estrés postraumático y cerebro. Revista de la Asociación Española de Neuropsiquiatría, 33(119), 511-523.
Tamayo, N., Rincón, C. J., de Santacruz, C., Bautista, N., Collazos, J., Gómez-Restrepo, C. (2016). Problemas mentales, trastornos del afecto y de ansiedad en la población desplazada por la violencia en Colombia, resultados de la Encuesta Nacional de Salud Mental 2015. Revista Colombiana de Psiquiatría, 45(1), 113-118.

Vieyra Moreno C. I., Gurrola Peña G. M., Balcazar Nava P., Bonilla Muñoz M. P. y Virseda Heras, J. A. (2009). Estado de salud mental en mujeres víctimas de violencia conyugal que acuden a la procuraduría general de justicia del estado de México. Psicología Iberoamericana, 17(1), 57-64.

White, S. F., Costanzo, M. E., Blair, J. R. y Roy, M. J. (2014). PTSD symptom severity is associated with increased recruitment of top-down attentional control in a trauma-exposed sample. Neurolmage: Clinical, 18(7), 19-27. https://doi. org/10.1016/j.nicl.2014.11.012 